\title{
Quasi-Static Position Calibration of the Galvanometer Scanner on the Additive Manufacturing Metrology Testbed
}

\author{
Brandon Lane \\ Shawn Moylan \\ Ho Yeung \\ Jorge Neira \\ Josephine Chavez-Chao
}

This publication is available free of charge from:

https://doi.org/10.6028/NIST.TN.2099

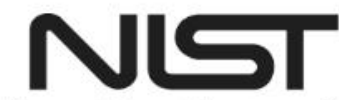

National Institute of Standards and Technology U.S. Department of Commerce 
NIST Technical Note 2099

\title{
Quasi-Static Position Calibration of the Galvanometer Scanner on the Additive Manufacturing Metrology Testbed
}

\author{
Brandon Lane \\ Shawn Moylan \\ Ho Yeung \\ Josephine Chavez-Chao \\ Intelligent Systems Division \\ Engineering Laboratory \\ Jorge Neira \\ Sensor Science Division \\ Physical Measurement Laboratory
}

This publication is available free of charge from:

https://doi.org/10.6028/NIST.TN.2099

June 2020

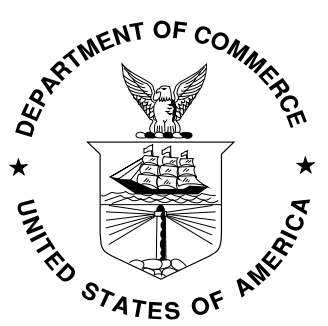

U.S. Department of Commerce

Wilbur L. Ross, Jr., Secretary

National Institute of Standards and Technology Walter Copan, NIST Director and Undersecretary of Commerce for Standards and Technology 
Certain commercial entities, equipment, or materials may be identified in this document in order to describe an experimental procedure or concept adequately.

Such identification is not intended to imply recommendation or endorsement by the National Institute of Standards and Technology, nor is it intended to imply that the entities, materials, or equipment are necessarily the best available for the purpose.

National Institute of Standards and Technology Technical Note 2099

Natl. Inst. Stand. Technol. Tech. Note 2099, 25 pages (June 2020)

CODEN: NTNOEF

This publication is available free of charge from:

https://doi.org/10.6028/NIST.TN.2099 


\begin{abstract}
The Additive Manufacturing Metrology Testbed (AMMT) is a fully customized laser powder bed fusion (LPBF) additive manufacturing (AM) research platform designed and constructed at the National Institute of Standards and Technology. This system incorporates a galvanometer (galvo) scanner, consisting of two mirrors which can be controlled to rotate over fixed angles and reflect and position a focused, high power laser onto a substrate. This Technical Note describes the methods for calibrating the quasi-static position of the laser spot, positioned via the galvo, as a function of the digital command to the galvo controller. In addition, the procedures for calibrating the galvo position encoders to the physical laser spot position are provided. An example is provided of measured galvo position errors before and after calibration and compensation. Finally, an error reporting and uncertainty budget is provided.
\end{abstract}

\title{
Disclaimer
}

The procedures outlined in this document are provided solely as reference to current methods employed on an experimental apparatus at NIST. Readers should note, these procedures reflect only the current methods employed by NIST, which may be further improved or amended. Readers should not interpret the procedures in this document as a standard or best practice nor refer to this document as such.

\section{Key words}

Additive Manufacturing, Calibration, Galvanometer, Laser Powder Bed Fusion. 


\section{Table of Contents}

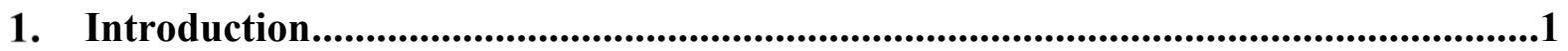

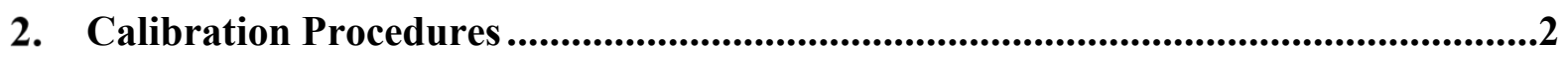

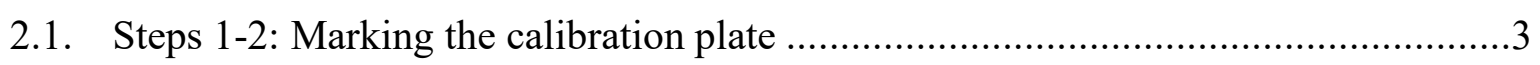

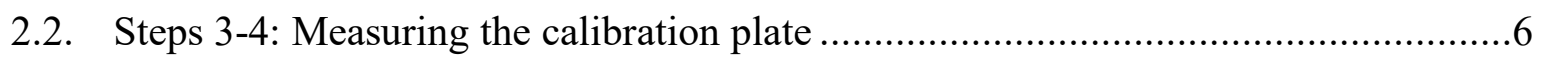

2.3. Steps 5-6: Calculating the calibration functions ..................................................

2.4. Step 7: Galvo Positioning Error Compensation.................................................12

3. Error Reporting and Example Calibration and Compensation Results....................12

4. Measurement Uncertainty ..........................................................................................15

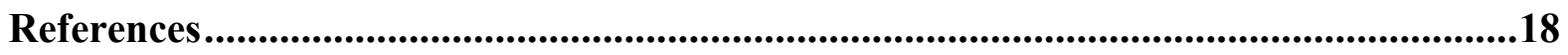

Appendix A: Supplemental Materials .....................................................................................19

\section{List of Tables}

Table 1: Measurement uncertainty budget for the mark and measure procedure. Uncertainty values are presented after any correction for non-normal (Gaussian) probability distribution.

\section{List of Figures}

Fig. 1: Schematic of the galvo system control flow and calibration functions for $\mathrm{X}$ positioning and $\mathrm{X}$ encoder readout (same schematic could be made for $\mathrm{Y}$ ) . ........................ 1

Fig. 2: Calibration plate put into AMMT, including enclosure with laser window..................3

Fig. 3: Screenshot of the 'calibration mode' on the AMMT controller. ................................4

Fig. 4: Positions and ordering of the $7 \times 7$ grid of mark marks executed in the AMMT galvo calibration routine.

Fig. 5: XY Calibration Routine virtual instrument front panel, showing the commanded positions (Xc, Yc) in [mm], digital positions (XD,YD) in digital levels, encoder voltages $(\mathrm{X}(\mathrm{V}), \mathrm{Y}(\mathrm{V}))$ in $[\mathrm{V}]$, and calculated encoder scaled positions (Xe, Ye) in [mm]. Note that at the time of publication, the column headers in the VI had not been changed to match those used throughout this document.

Fig. 6: Example scanned image of a laser mark. (a) Scanned image of full calibration plate.

(b) Subset of (a) showing four laser marks. (c) Microscope image of the lower-left mark in

(b) with $50 \mu \mathrm{m}$ grid overlay. (d) Resulting image from flatbed scanner of the same mark ......6

Fig. 7: Steps for measuring dots on the calibration plate. a) Example setting the image scale.

b) Main window displaying the scanned plate and ROIs. c) Magnified image showing a circular ROI around a mark. d) List of ROIs in the ROI manager. e) Example CoM measurement results table.

Fig. 8: Example section of the calibration calculation spreadsheet, including what each column data represents and how it is obtained.

Fig. 9: Best fit line through $(\mathrm{x}, 0)$ measured points. Y-offset $\left(\mathrm{y}_{0}\right)$ is found to be $0.0389 \mathrm{~mm}$, and rotation angle $\theta=0.0618 \mathrm{rad}$. 
Fig. 10: Results of shifting by $\mathrm{y}_{0}$ and rotating by $\theta$ moves center horizontal points $(\mathrm{x}, 0)$ to align with ${ }^{\mathrm{A}} \mathrm{y}=0$ origin.

Fig. 11: Best-fit line through central vertical points, $(0, y)$, after translating to $\mathrm{y}_{0}$, and rotating by $\theta$. The intercept defines the $\mathrm{x}$-offset, $\mathrm{x}_{0}$. Note that the $\mathrm{x}$ and $\mathrm{y}$ axes are switched in this plot.

Fig. 12: Results of shifting by $\mathrm{x} 0$ on the central vertical points $(0, \mathrm{y})$. The intercept of the best-fit line is now at ${ }^{\mathrm{A}}(0,0)$.

Fig. 13: Galvo calibration functions determined from mapping the measured points translated into machine coordinates ${ }^{\mathrm{A}}(\mathrm{x}, \mathrm{y})$ in $[\mathrm{mm}]$ to the digital command $\left(\mathrm{x}_{\mathrm{cmd}}, \mathrm{y}_{\mathrm{cmd}}\right)$ in $[\mathrm{DL}]$. .11 Fig. 14: Galvo encoder calibration functions determined from mapping the measured points in machine coordinates ${ }^{\mathrm{A}}(\mathrm{x}, \mathrm{y})$ in $[\mathrm{mm}]$ to the measured encoder voltage levels ( $\mathrm{x}_{\text {enc }}, \mathrm{Y}_{\mathrm{enc}}$ ) in [V] from the DAQ data.

Fig. 15: Example commanded points (+), measured points after rotation (o), and the vector difference between them (scaled $\times 20$ ) for pre-compensated and post-compensated measurements, demonstrating improvement in positioning accuracy post-compensated.......12 Fig.16: Example errors $\mathrm{E}_{\mathrm{x}}$ and $\mathrm{E}_{\mathrm{y}}$ before compensation (left) and after compensation (right). After compensation various error metrics are reduced, as shown in the tables at the bottom of each figure.

Fig. 17: Top: Example heat map of the vector sum error from pre- and post- compensation measurements, fit with a $4^{\text {rd }}$ order two-dimensional polynomial, and sub-sampled to a $1 \mathrm{~mm}$ grid. Bottom: Cumulative distributions of the sub-sampled vector-sum errors $|\mathrm{E}|$. .15 Fig. 18: Example ImageJ window showing 31 circular ROIs selected to test how varying the user-selected ROI size and location will affect the calculation of the spot's CoM. .17 


\section{Introduction}

The Additive Manufacturing Metrology Testbed (AMMT) is a fully functioning laser powder bed fusion (LPBF) research platform, which includes a myriad of in-situ sensors and instrumentation for studying LPBF process physics. The AMMT has fully customized controls that utilizes a 2D galvanometer (galvo) based laser steering system consisting of two rotating mirrors with approximately orthogonal axes, which position the focused laser spot at specified locations in $\mathrm{X}$ and $\mathrm{Y}$ coordinates on the build plane. Yeung et al. provides more details on the controller implementation and characterization of various aspects including dynamic performance, synchronization with other AMMT systems, and complex laser/galvo positioning control $[1,2]$. Additionally, Yeung et al. described an experimental in-situ method for calibrating the position of the laser/galvo system which has potential for automation and more rapid calibrations [3]. However, at the time of publication of this document, that system has not been developed beyond the experimental stage.

This document describes the methods for calibrating and compensating ${ }^{1}$ the positioning errors of the laser galvo system via the AMMT controller, and aims to accomplish the following:

1) Accurately position a focused laser spot on the build plane at specified or commanded locations provided to the AMMT controller

2) Calibrate the galvo encoders to read out the accurate laser spot position in length units

3) Through the calibration process, establish the machine coordinate system's $X$ and $\mathrm{Y}$ axes and origin

4) Define metrics for quantifying and comparing galvo positioning errors

5) Demonstrate the results of an example calibration and compensation procedure

A schematic of the AMMT galvo control system is shown in Fig. 1, which demonstrates the conversion of commanded scan positions to final, physical scan positions. These conversions depend on user-determined calibration functions, which are incorporated into the digital galvo control system, or the electromechanical system itself.

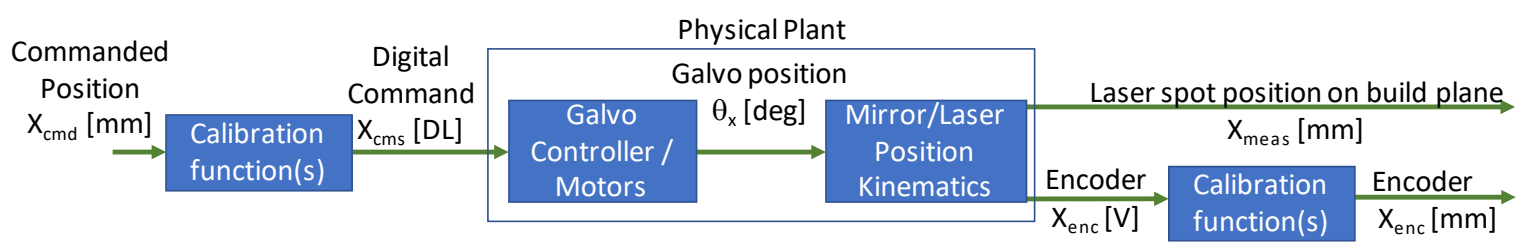

Fig. 1: Schematic of the galvo system control flow and calibration functions for $\mathrm{X}$ positioning and $\mathrm{X}$ encoder readout (same schematic could be made for $\mathrm{Y}$ ).

The general goal of the galvo calibration is to measure the quasi-static positioning errors, then define and apply compensation functions that mimic the physical plant shown in Fig. 1,

\footnotetext{
${ }^{1}$ The terms for 'calibration' and 'compensation' used here are based on terminology. The International Vocabulary of Metrology (VIM) [4], recommends 'correction' instead of 'compensation, While the VIM principally describes metrology systems (values are read from and instrument), the terms are also applied here to the control system (values are sent to an instrument to execute).
} 
and equates $X_{\text {meas }}=X_{c m d}$, or $X_{\text {meas }}=X_{\text {enc. }}$ For the galvo position command, this is done by creating physical marks with the laser on a substrate, and measuring their physical geometry or position. This procedure is colloquially called the 'mark and measure' procedure [5]. Comparing the commanded galvo positions defined in digital levels [DL], and the measured positions enables construction of calibration functions $X_{D L}[D L]=f\left(X_{\text {meas }}[\mathrm{mm}]\right)$, which essentially mimic the 'Physical Plant' shown in Fig. 1, and are used to then convert commanded physical position $\mathrm{X}_{\mathrm{cmd}}$ in millimeters [mm] into the galvo digital commands $\mathrm{X}_{\mathrm{DL}}$. . This practice can be used to both develop new calibration functions, or measure positioning errors of a current calibration. Similarly, galvo encoders provide an analog output voltage that can be read by a data acquisition (DAQ) system, an related to the galvo position. The mark and measure process can be used to generate a calibration function $\mathrm{X}_{\mathrm{enc}}$ $[\mathrm{mm}]=\mathrm{f}\left(\mathrm{X}_{\mathrm{enc}}[\mathrm{V}]\right)$, which converts measured encoder position in volts [V] into physical position in millimeters $[\mathrm{mm}]$. The encoder signals are then useful to determine if there is any following error between the commanded position $\mathrm{X}_{\mathrm{cmd}}$ and the real positions executed by the galvo and galvo controller.

Multiple methods and strategies exist for positional calibration of a laser-galvo system, some of which would likely result in an improvement in precision from the procedure outlined here. These typically employ a 'mark and measure' approach, where the laser is steered to create marks on a substrate (typically either coated glass [5] or anodized metal $[6,7])$, then the geometry or positions of those marks, are measured using an offline dimensional measuring system to establish the galvo positioning errors and any error compensation necessary. While in-situ methods have been demonstrated on the AMMT [3], the general 'mark and measure' concept is demonstrated here by creating physical marks, then measuring their true position against some external dimensional scale, which achieves sufficient level of galvo relative positioning accuracy required by the AMMT. This required relative accuracy is generally defined as better than $10^{-3} \mathrm{~mm} / \mathrm{mm}$ (e.g., accurate positioning within $100 \mu \mathrm{m}$ over a $100 \mathrm{~mm}$ span), or $<0.1 \%$. Note that for commercial systems making production parts on much larger build areas $\left(250^{2} \mathrm{~mm}^{2}\right.$ is typical, compared to AMMT's $100^{2} \mathrm{~mm}^{2}$ ), the positioning accuracy requirements are likely more stringent.

\section{Calibration Procedures}

The following lists the general steps in the AMMT galvo calibration procedure:

1) Obtain a $100 \mathrm{~mm}$ by $100 \mathrm{~mm}$ sheet of black anodized aluminum plate, and place it in the AMMT build chamber, ensuring the z-height position is at the defined build plane height $(0.70 \mathrm{~mm}$ above the reference mark).

2) Turn on a flow of argon, close the AMMT build chamber, and execute the "Calibration Mode" on the AMMT controller to mark a dot grid pattern on the anodized plate. Collect the galvo digital command values and galvo encoder signal values as part of the "DAQ file" while performing the mark.

3) Remove the marked dot grid and scan it in a calibrated flatbed scanner (the external dimensional scale) at a high resolution (4800 dots per inch, or $5.3 \mu \mathrm{m}$ per pixel).

4) Using Image J software, measure the center of mass (CoM) locations for each dot.

5) Begin formulating the calibration spreadsheet, and orient and translate the measured dot mark positions to establish the machine $\mathrm{X}$ and $\mathrm{Y}$ coordinates. 
6) Finish the calibration spreadsheet by adding galvo encoder values extracted from the DAQ file, and calculate the calibration functions and calibration error.

7) Optional: Apply the new galvo position calibration functions back into the AMMT controller, and repeat steps 1-6 to measure the residual galvo positioning error.

\subsection{Steps 1-2: Marking the calibration plate}

Select a black-anodized 5005 aluminum plate (McMaster-Carr ${ }^{2}$ Part\# 6062K1), cut to approximately $100 \mathrm{~mm}$ by $100 \mathrm{~mm}$. Mark the plate with the date and arrows indicating the nominal $\mathrm{X}$ and $\mathrm{Y}$ orientation. Place it in the AMMT build well, and measure the plate using the recoater-mounted profilometer. First, position the profilometer spot adjacent to the Zheight reference mark (a small indent approximately $1 \mathrm{~cm}$ to the left of the build well) and zero the profilometer, or note the indicated position. Then move the profilometer over the approximate center of the calibration plate and position the build plate mechanism via the AMMT controller until the calibration plate is $0.70 \mathrm{~mm}$ above the $\mathrm{z}$-height reference mark (see Fig. 2). This position ( $0.70 \mathrm{~mm}$ above the reference mark) has been established by the AMMT designers as the 'build plane height', at which many other measurements are taken or the recoater blade is set prior to a 3D build. Ensure an enclosure with gas connection and clean laser window is in place. Run approximately $20 \mathrm{~L} / \mathrm{min}$ of argon gas into the enclosure.

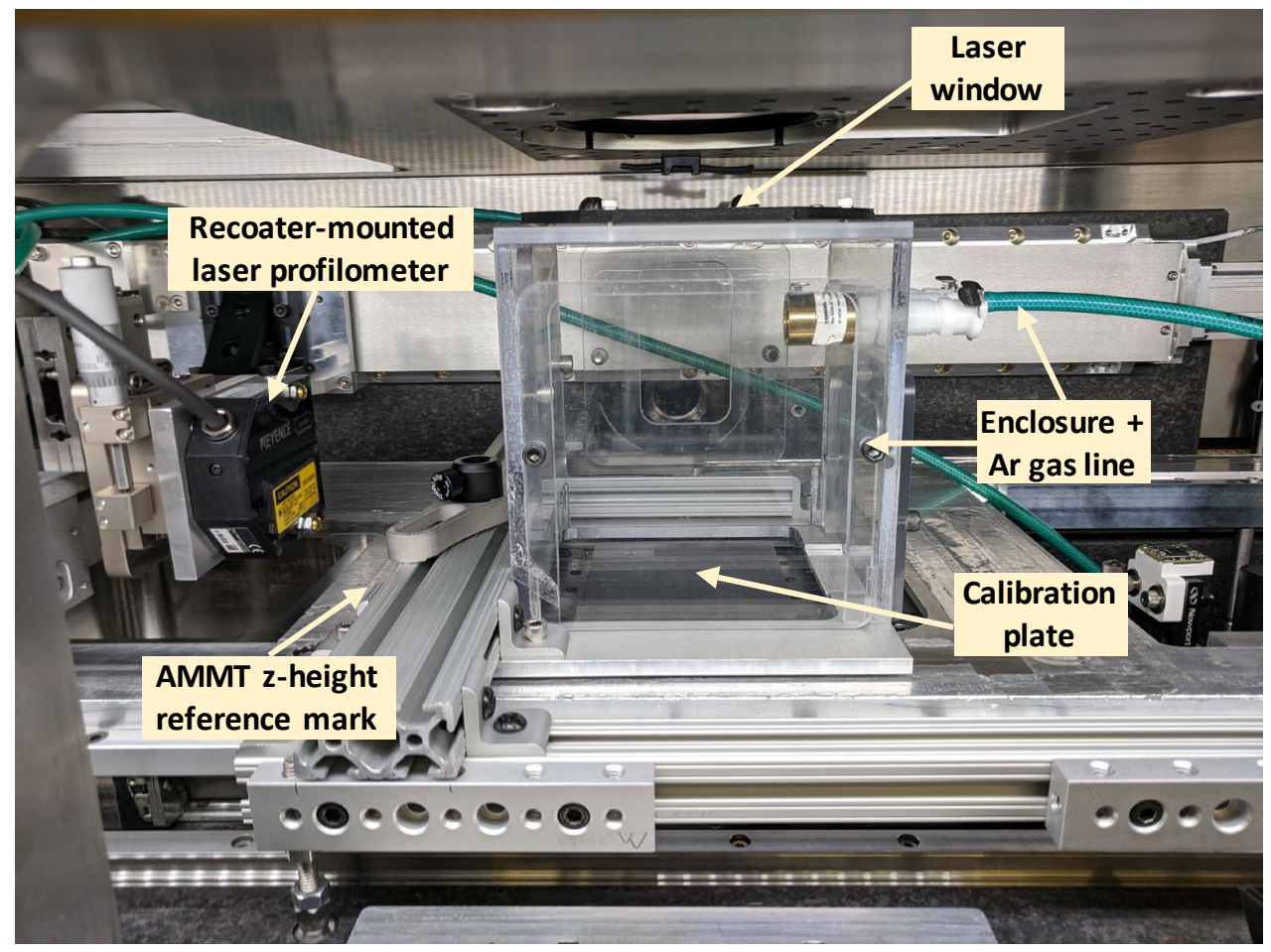

Fig. 2: Calibration plate put into AMMT, including enclosure with laser window.

\footnotetext{
${ }^{2}$ Certain commercial equipment, instruments, or materials are identified in this paper in order to specify the experimental procedure adequately. Such identification is not intended to imply recommendation or endorsement by the National Institute of Standards and Technology, nor is it intended to imply that the materials or equipment identified are necessarily the best available for the purpose.
} 
Close the AMMT chamber. Start the 'Calibration Mode' in the controller software, as shown in Fig. 3, under 'Advanced Tools' menu. This adds several buttons and indicators to the main AMMT controller front panel, including a large indicator light in the lower left to show the system is in galvo calibration mode. Ensure the laser is engaged (E-stop off, laser console green button 'on', red warning light flashing). Click 'execute' to fire the laser and mark the plate.

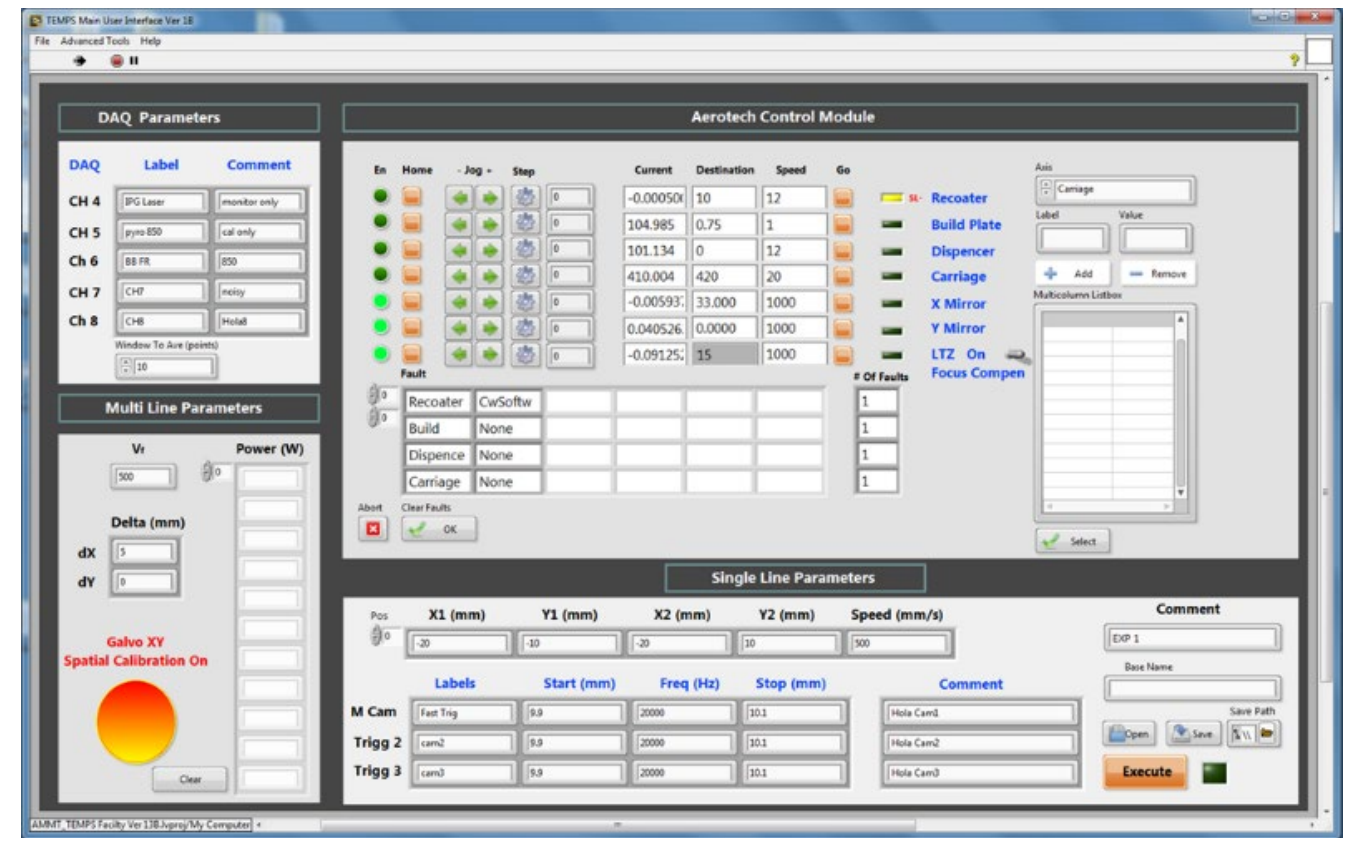

Fig. 3: Screenshot of the 'calibration mode' on the AMMT controller.

A series of points will be marked with the laser as shown in Fig. 4, with the laser turned off while traversing from point to point. Each point consists of the laser holding a static position for $100 \mathrm{~ms}$ at approximately $15 \mathrm{~W}$ power. Note that one of the points near the end is missing. This helps ensure that the $\mathrm{XY}$ alignment of the dot pattern is distinguishable when the calibration plate is removed from the chamber. The AMMT data acquisition (DAQ) system incorporates multiple analog to digital (A/D) converter channels. Two of these channels sample the voltage output signal from the galvo encoders at $100 \mathrm{kHz}$. During the calibration scan, the DAQ should have recorded the galvo encoder signals, as well as any other connected channels, and saved them on the AMMT controller. 


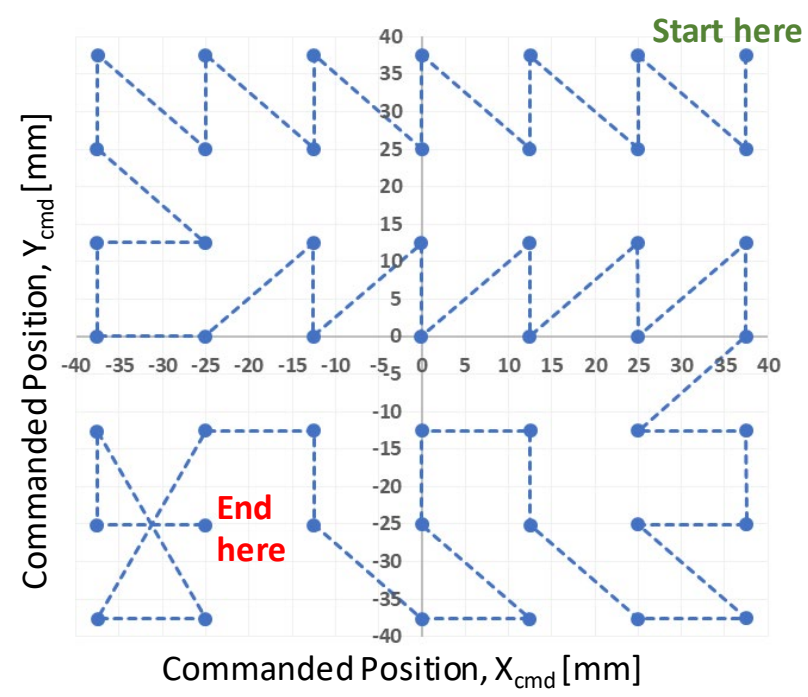

Fig. 4: Positions and ordering of the $7 \times 7$ grid of mark marks executed in the AMMT galvo calibration routine.

The galvo encoder positions, collected as timeseries data, needs to be converted into average values in $[\mathrm{V}]$ over the duration that the laser is marking each point. This is done by running the separate 'XY Calibration Routine.vi' virtual instrument (VI) shown in Fig. 5, which performs this averaging and exports the required data in a CSV file. Run the 'XY Calibration Routine' VI, and supply it the DAQ file that was generated during the scan. Save the generated CSV file.

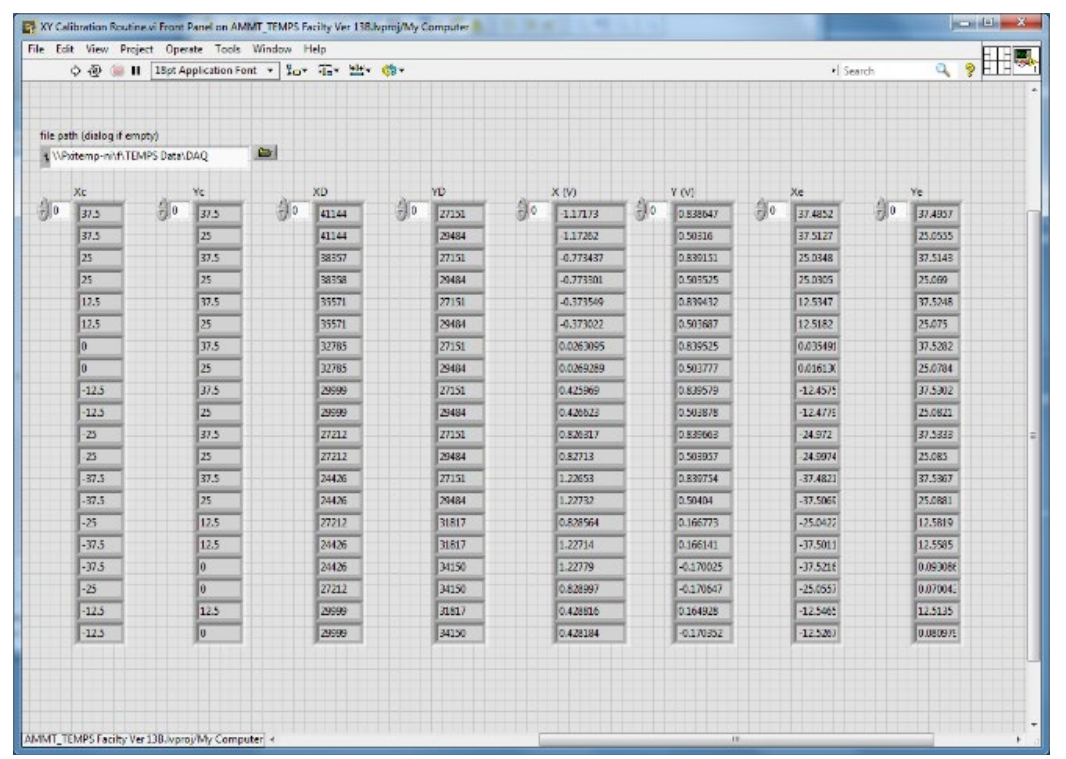

Fig. 5: XY Calibration Routine virtual instrument front panel, showing the commanded positions (Xc, Yc) in [mm], digital positions (XD,YD) in digital levels, encoder voltages $(\mathrm{X}(\mathrm{V}), \mathrm{Y}(\mathrm{V}))$ in $[\mathrm{V}]$, and calculated encoder scaled positions (Xe, Ye) in [mm]. Note that at the time of publication, the column headers in the VI had not been changed to match those used throughout this document. 


\subsection{Steps 3-4: Measuring the calibration plate}

Carefully remove the calibration plate from the AMMT being careful not to scratch or leave fingerprints, and wrap it in a laboratory wipe for safe transport to the flatbed scanner. Carefully place the calibration plate face-down on the flatbed scanner. The pixel pitch of the flatbed scanner is used as the dimensional reference scale for measuring the marked dots. The accuracy of this scale was verified against a calibrated target, described as the Scaling Factor in the uncertainty assessment in Section 4. In the scanner software, set the image type to 8-bit grayscale, and 4800 dots per inch. Use the 'Preview' tool to run a quick (low resolution) scan. In the preview window, use the marquee tool to draw a rectangle around the calibration grid, which reduces the area over which the scan is performed. This should generate an approximately 19000 pixel by 19000 pixel image of 350 Megabytes. An example of a scanned image is shown in Fig. 6.
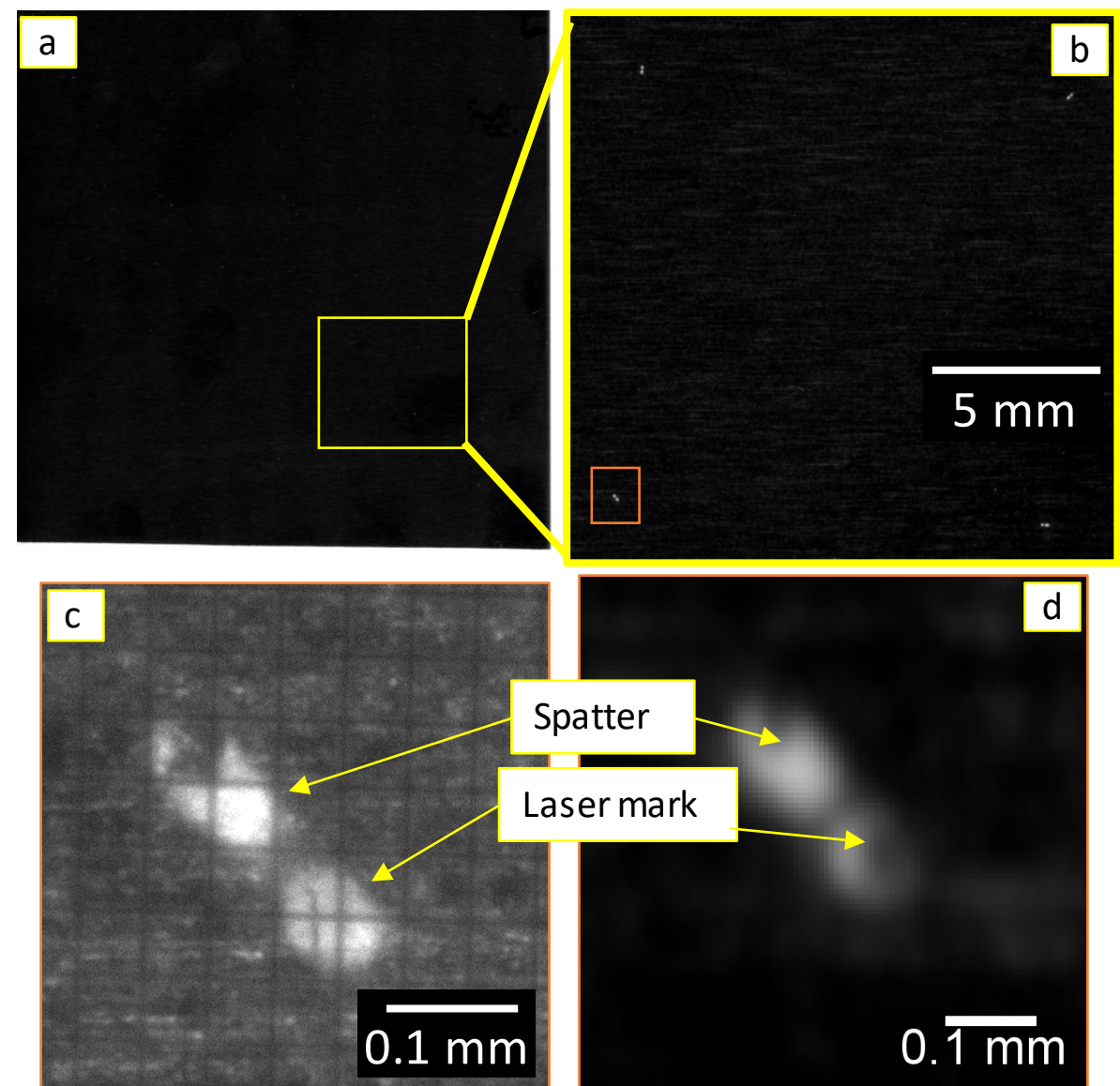

Fig. 6: Example scanned image of a laser mark. (a) Scanned image of full calibration plate. (b) Subset of (a) showing four laser marks. (c) Microscope image of the lower-left mark in (b) with $50 \mu \mathrm{m}$ grid overlay. (d) Resulting image from flatbed scanner of the same mark

Note that some of the laser marks may incur a deposit of spatter, which is nominally oriented in the direction the laser was scanning toward the point, as shown in Fig. 4. 
The following steps are done to measure the locations of the marks after opening the image in ImageJ.

1) Adjust the approximate origin of the image to the central dot: Zoom in to the central dot using the (+) and (-) keys, and note the visually approximated center in pixels (listed under the ImageJ main window). Under Image $>$ Properties, set the origin to these values. This reduces the offsets needed to later center and align the measured points, and allows the user to better identify if the scaling is as expected.

2) Apply scaling: Under Analyze>Set scale, adjust the scaling to be 188.9764 pixels per $\mathrm{mm}$ (equivalent to 4800 dots per inch).

3) Create list of regions of interest (ROIs) around mark points: Using the circle ROI tool, draw a circle around one of the marks, using (+) or (-) keys to zoom in and out. Once the first ROI is made, type ' $t$ ' to add it to a list in the ROI Manager. Repeat for all the marks.

4) Measure the centers of mass: Under Analyze>Set Measurements..., click on 'Center of Mass'. Click on 'Invert Y coordinates' to ensure the image coordinate origin is in the lower left, then close the window. Click 'Measure' on the ROI manager, and a table of measurement results will appear with the CoM within each ROI listed as shown in Fig. 7. Save this table.

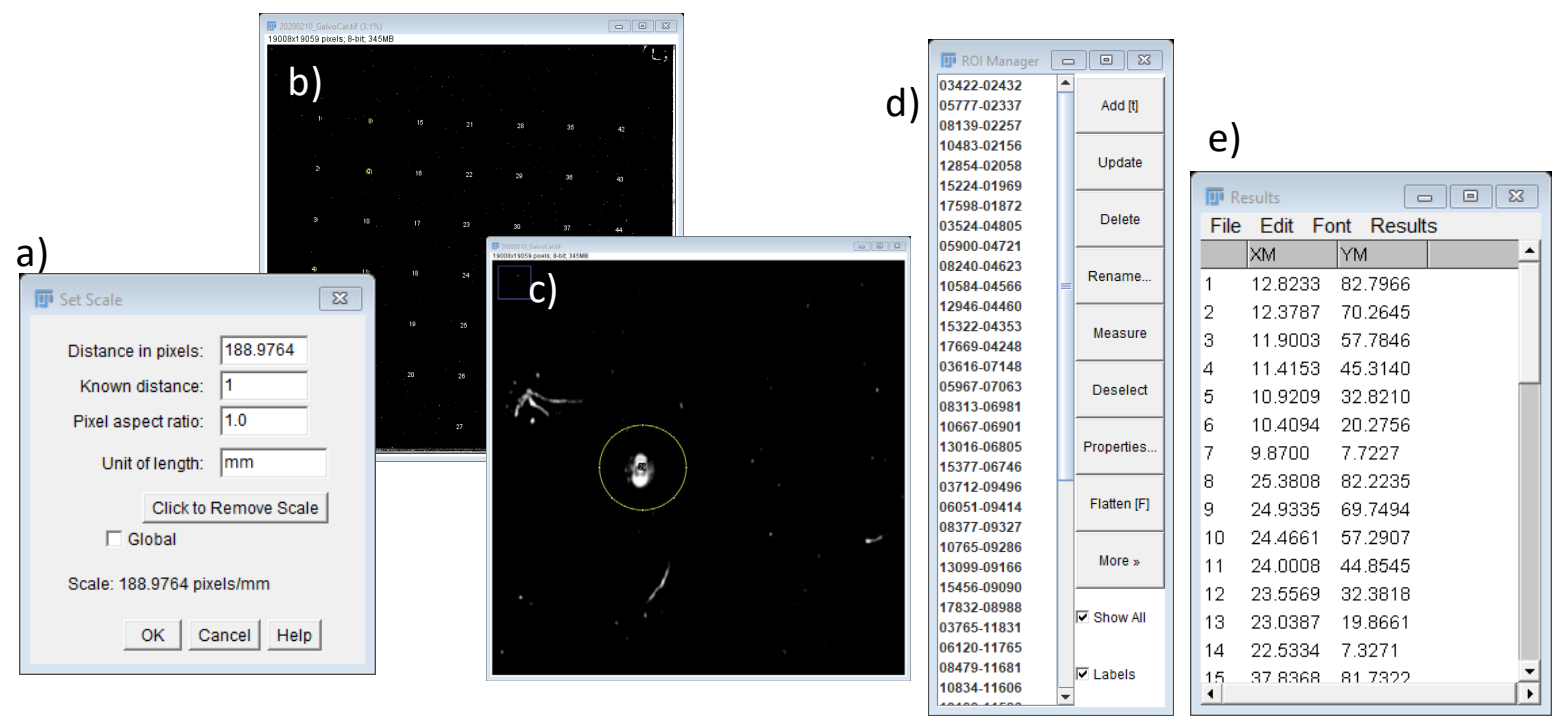

Fig. 7: Steps for measuring dots on the calibration plate. a) Example setting the image scale. b) Main window displaying the scanned plate and ROIs. c) Magnified image showing a circular ROI around a mark. d) List of ROIs in the ROI manager. e) Example CoM measurement results table.

\subsection{Steps 5-6: Calculating the calibration functions}

Calculations are conducted using commercial spreadsheet calculation software, although any calculation and plotting software is sufficient. Arrays are copied from their respective sources (e.g., measured points or DAQ data), and put into columns in the spreadsheet as shown in Fig. 8. First, the target commanded scan positions, as shown in Fig. 4, are copied to the first two columns. It is important to keep the ordering of the XY pairs consistent between data sources. This can be specified in Excel $^{1}$ by clicking 
Sort \& Filter $>$ Custom Sort..., then sorting X column smallest to largest, followed by Y column largest to smallest. This should also be done for the encoder measurements from the DAQ output file and the scanner measurements prior to copying to the main calculation sheet.

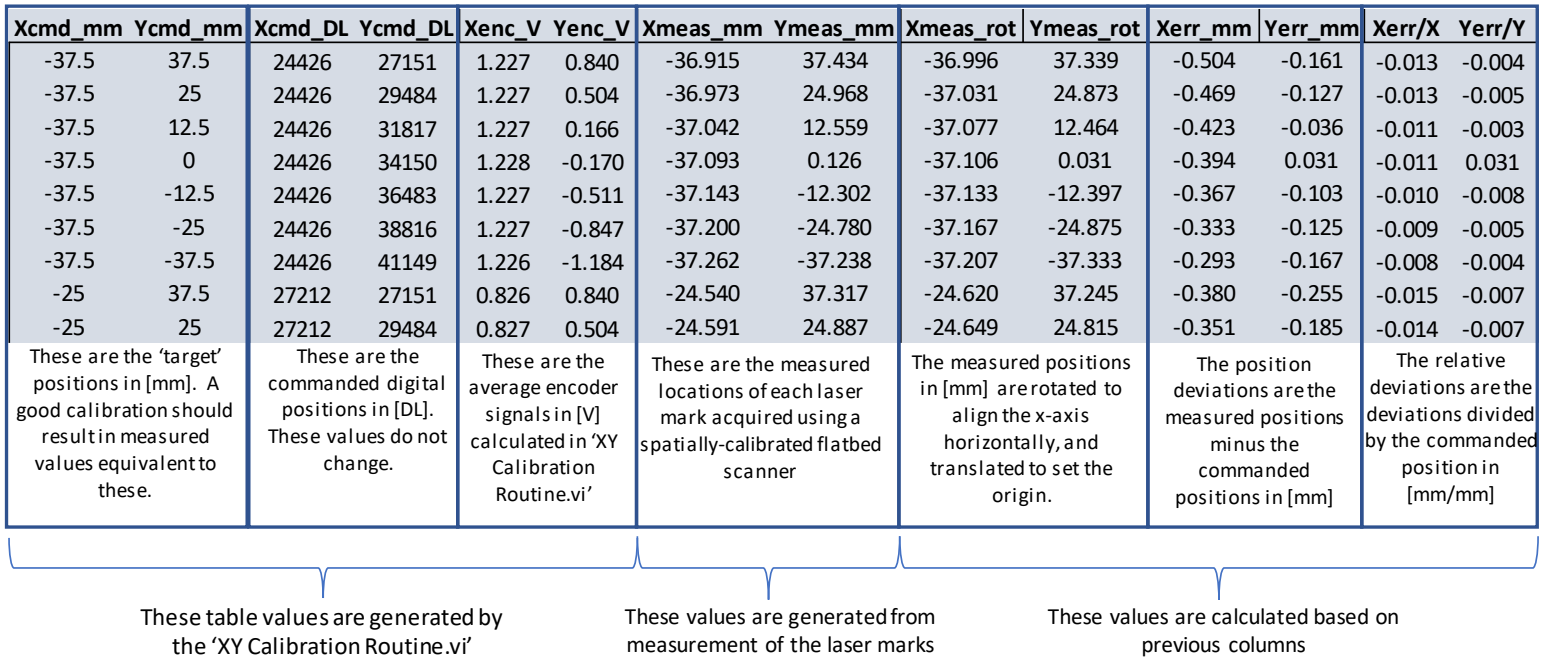

Fig. 8: Example section of the calibration calculation spreadsheet, including what each column data represents and how it is obtained.

Since the relative orientation of the calibration plate with respect to the galvo position is lost when the plate is removed from the machine, the measured points will need to be oriented and translated to coincide with the definition of the machine $\mathrm{X}$ and $\mathrm{Y}$ axes (defined in detail below). Machine axes are indicated by the superscript ' $A$ ' to differentiate from other axes. To rotate and center the grid pattern of the measured points, the measured points along the center horizontal row are selected, which coincide with $X$-values when $\mathrm{y}_{\mathrm{cmd}}=0[\mathrm{~mm}]$. A regression line is then calculated through these points, as shown in Fig. 9. The y-offset, $\mathrm{y}_{0}$, is identified as the $y$-intercept, and rotation angle $\theta$ in radians [rad] as the slope.

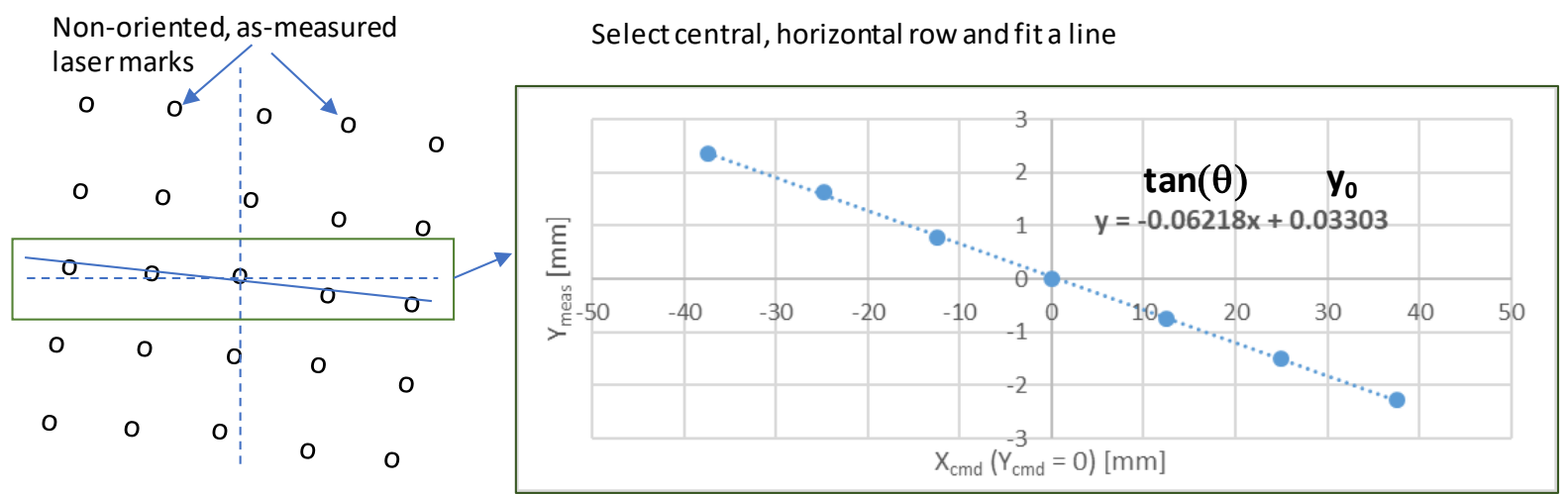

Fig. 9: Best fit line through $(\mathrm{x}, 0)$ measured points. Y-offset $\left(\mathrm{y}_{0}\right)$ is found to be $0.03303 \mathrm{~mm}$, and rotation angle $\theta=\operatorname{atan}(0.06218)=0.06210 \mathrm{rad}$. 
The measured points are then shifted by $y_{0}$ in the calculation spreadsheet to better align with the anticipated origin, then rotated based on Eqn. (1). Note that points have not yet been centered in $X$, indicated by the asterisk on $\mathrm{X}_{\text {meas,rot }}$ * in Eqn. (1). Results of the translation by $\mathrm{y}_{0}$, and rotation by $\theta$ are shown in Fig. 10, which shows the best fit line through the points $(\mathrm{x}, 0)$ now closely intercept $\mathrm{y}=0$.

$$
\left\{\begin{array}{c}
x_{\text {meas }, \text { rot }} * \\
y_{\text {meas }, \text { rot }}
\end{array}\right\}=\left|\begin{array}{cc}
c(\theta) & -s(\theta) \\
s(\theta) & c(\theta)
\end{array}\right|\left\{\begin{array}{c}
x_{\text {meas }} \\
y_{\text {meas }}-y_{0}
\end{array}\right\}_{y c m d=0}
$$

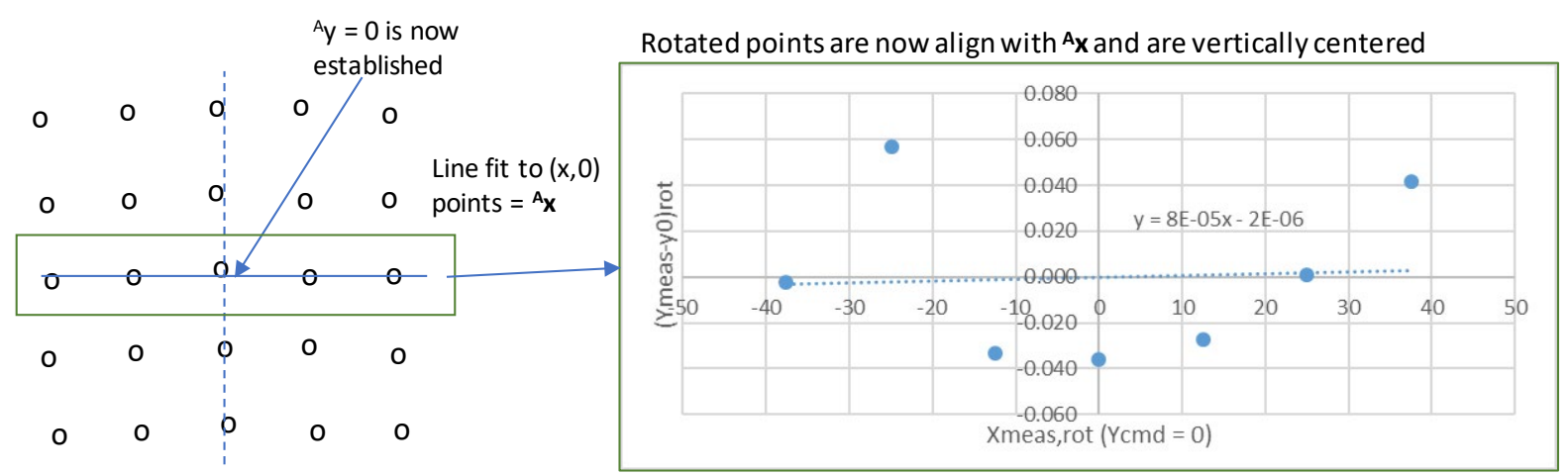

Fig. 10: Results of shifting by $\mathrm{y}_{0}$ and rotating by $\theta$ moves center horizontal points $(\mathrm{x}, 0)$ to align with ${ }^{\mathrm{A}} \mathrm{y}=0$ origin.

The machine $\mathrm{x}$-axis, ${ }^{\mathrm{A}} \mathbf{x}$, is now established, as well as the origin point ${ }^{\mathrm{A}} \mathrm{y}=0$. The $\mathrm{x}$-offset, $\mathrm{x}_{0}$, is then determined by fitting a line to the central vertical points, $(0, \mathrm{y})$, as shown in Fig. 11.

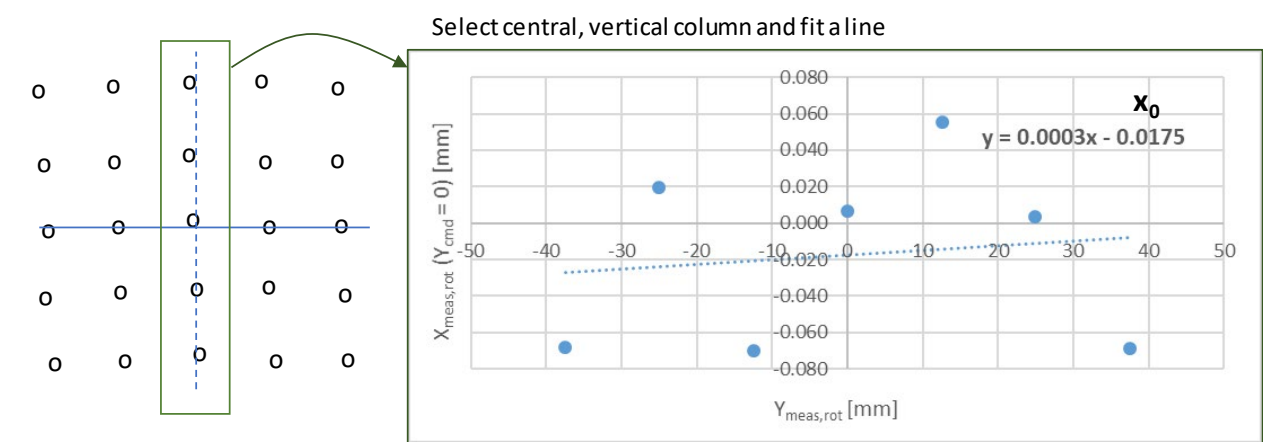

Fig. 11: Best-fit line through central vertical points, $(0, y)$, after translating to $\mathrm{y}_{0}$, and rotating by $\theta$. The intercept defines the $\mathrm{x}$-offset, $\mathrm{x}_{0}$. Note that the $\mathrm{x}$ and $\mathrm{y}$ axes are switched in this plot.

This fit line may have a non-zero slope, which indicates the measured positions along this column of laser marks are non-orthogonal to ${ }^{A} \mathbf{x}$. Finally, the points are translated one more time based on the measured $\mathrm{x}$-offset, $\mathrm{x}_{0}$, to arrive at the final oriented and centered points, descried in Equation (2), with final results shown in Fig. 12.

$$
\left\{\begin{array}{l}
x_{\text {meas }, \text { rot }} \\
y_{\text {meas }, \text { rot }}
\end{array}\right\}=\left\{\begin{array}{c}
x_{\text {meas,rot }} *-x_{0} \\
y_{\text {meas }, \text { rot }}
\end{array}\right\}
$$




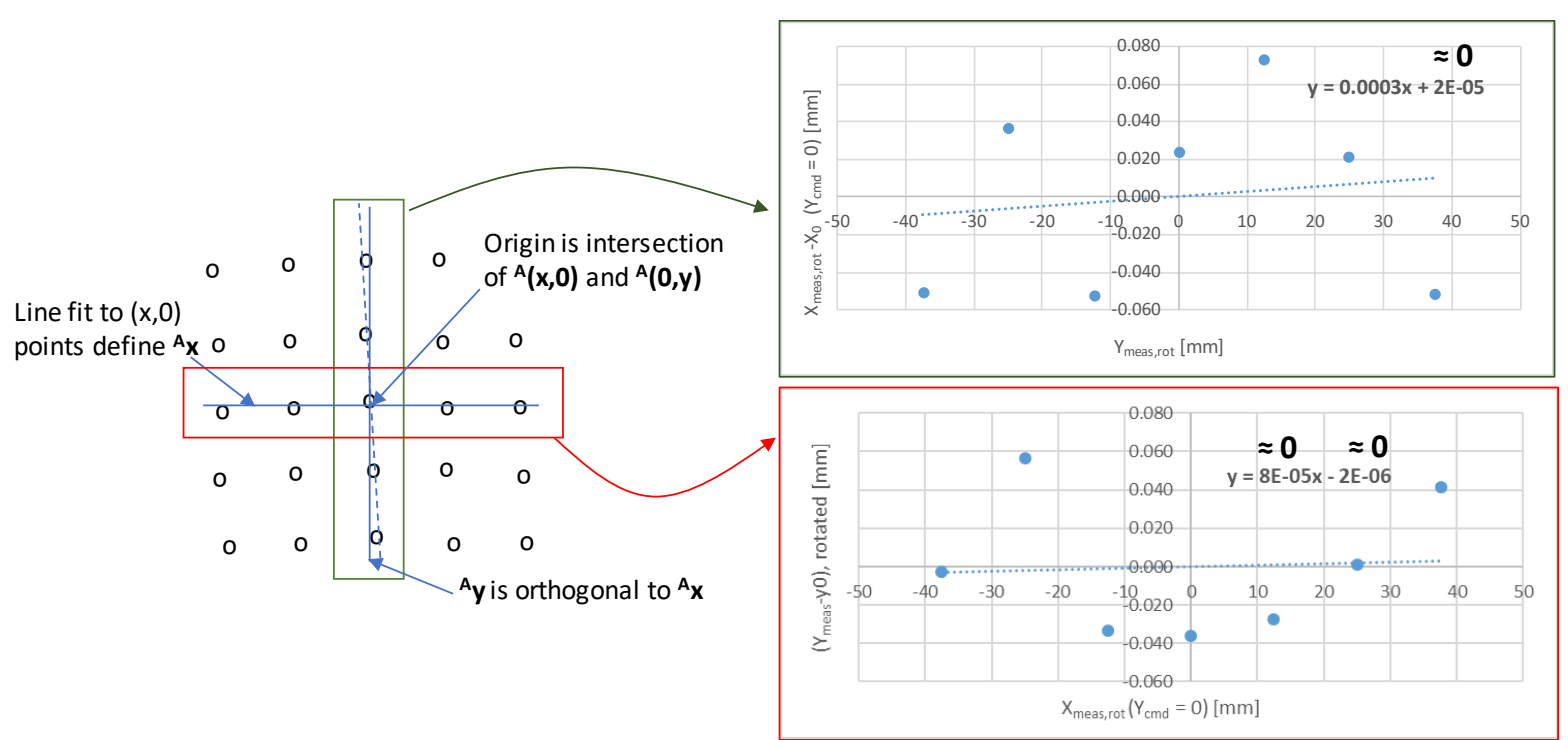

Fig. 12: Results of shifting by $\mathrm{x} 0$ on the central vertical points $(0, \mathrm{y})$. The intercept of the best-fit line is now at ${ }^{\mathrm{A}}(0,0)$.

The measured points along the central horizontal are now aligned with and define the machine $\mathrm{x}$-axis ${ }^{\mathrm{A}} \mathbf{x}$. The fit line through the central vertical points determines the origin ${ }^{A}(0,0)$ by its intersection with ${ }^{A} \mathbf{x}$, although it does not define the machine $y$-axis ${ }^{A} \mathbf{y}$. The machine $y$-axis ${ }^{A} \mathbf{y}$ is defined as being orthogonal to ${ }^{A} \mathbf{x}$ and passing through ${ }^{A}(0,0)$. Deviation of the fit line through the central vertical points from ${ }^{\mathrm{A}} \mathbf{y}$ indicates non-orthogonality error in the galvo system. After all measured points are oriented and translated to the machine coordinates (xmeas,rot, ymeas,rot), they can be considered ${ }^{\mathrm{A}}(\mathrm{x}, \mathrm{y})$.

Once the data in Fig. 8 is compiled (excluding the error measurements, which are described in the next section), the calibration functions for the galvo positioning (Fig. 13) and galvo encoders (Fig. 14) can then be calculated using linear regression. 

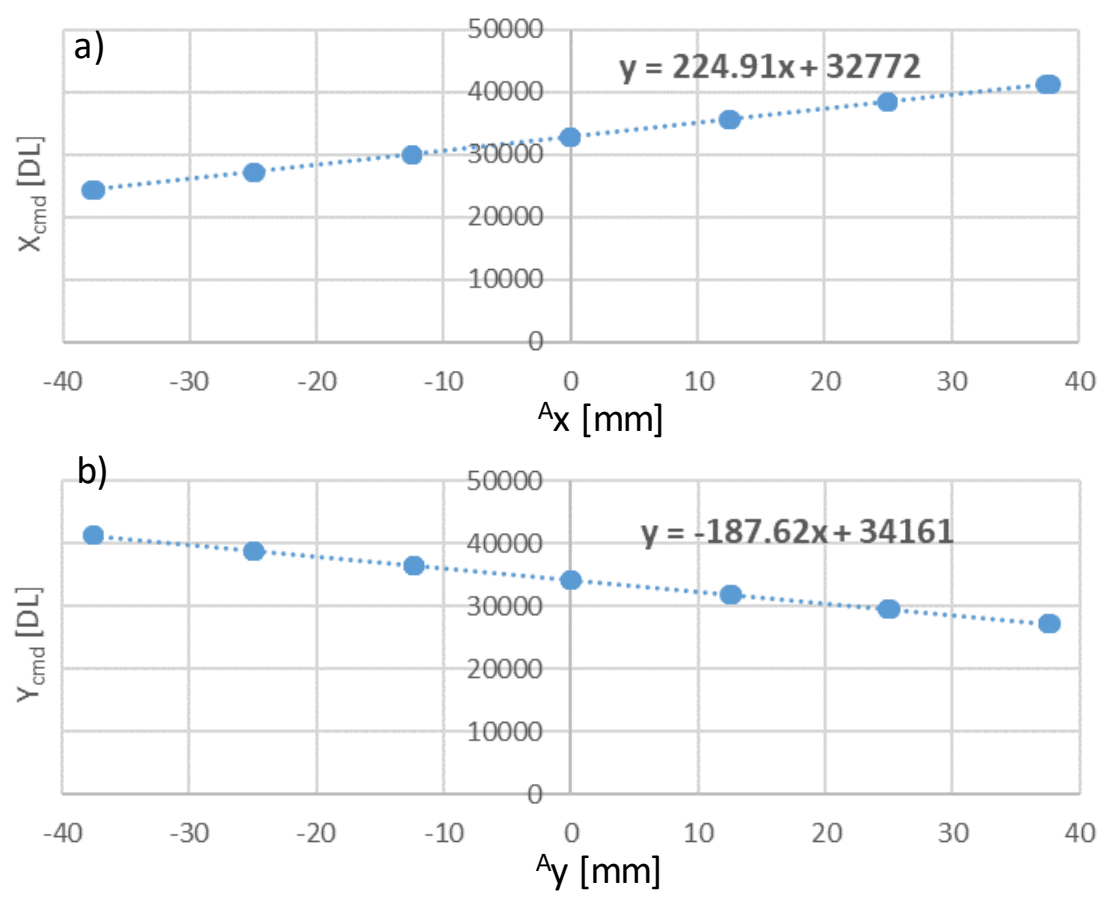

Fig. 13: Galvo calibration functions determined from mapping the measured points translated into machine coordinates ${ }^{\mathrm{A}}(\mathrm{x}, \mathrm{y})$ in $[\mathrm{mm}]$ to the digital command $\left(\mathrm{x}_{\mathrm{cmd}}, \mathrm{y}_{\mathrm{cmd}}\right)$ in $[\mathrm{DL}]$.

a)

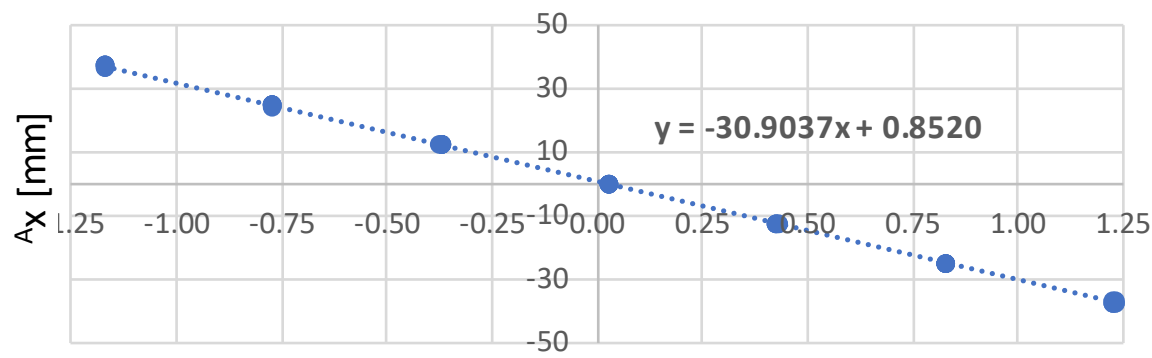

$$
\mathrm{X}_{\mathrm{enc}, \mathrm{V}}[\mathrm{V}]
$$

b)

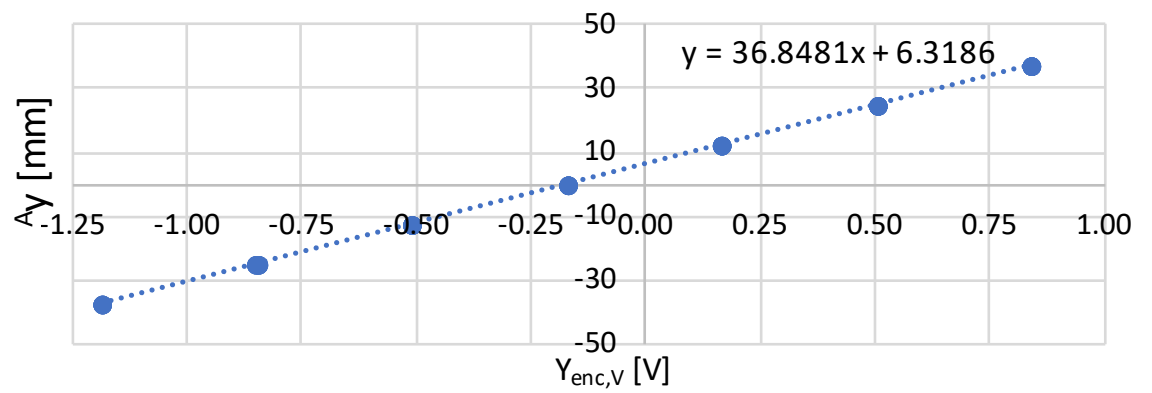

Fig. 14: Galvo encoder calibration functions determined from mapping the measured points in machine coordinates ${ }^{\mathrm{A}}(\mathrm{x}, \mathrm{y})$ in $[\mathrm{mm}]$ to the measured encoder voltage levels $\left(\mathrm{x}_{\mathrm{enc}}, \mathrm{y}_{\mathrm{enc}}\right)$ in $[\mathrm{V}]$ from the DAQ data. 
Note that in the AMMT main front panel, the encoder positions are read out in real time. Sometimes, a non-zero offset is incurred in the encoder readout in [V], which can vary over time, causing a non-zero offset in [mm] to be read from the encoders. If when the laser is commanded to move to a static position at the origin $(0,0)$ and the encoders read some nonzero offset, you can add or subtract that value in [mm] from the encoder calibration function. Since the encoder calibration equations in Fig. 14 are linear, this does not affect the scaling.

\subsection{Step 7: Galvo Positioning Error Compensation}

Once the galvo positioning calibration functions are calculated, such as those in Fig. 13, the slope and offset values from the calibration curve fits can be entered back into the AMMT controller. After this is completed, steps 1 to 6 should be repeated, and the physical position of the laser marks measured once again. If done correctly, this should reduce the difference between the commanded positions $\left(\mathrm{X}_{\mathrm{cmd}}\right.$ and $\left.\mathrm{Y}_{\mathrm{cmd}}\right)$ in $[\mathrm{mm}]$, and the measured positions $\mathrm{A}(\mathrm{x}, \mathrm{y})$ in $[\mathrm{mm}]$.

To verify that the correction has improved the galvo calibration, several metrics for calibration error are defined in the next section, which can be used to verify any improvement to the galvo calibration stemming from an applied correction.

\section{Error Reporting and Example Calibration and Compensation Results}

One component of the positioning error, which is the error between the true and commanded positions, is the vector difference between the corrected locations ${ }^{A}(x, y)$ and the commanded positions $(\mathrm{x}, \mathrm{y})_{\mathrm{cmd}}$. These error vectors can be plotted as a quiver plot, as in Fig. 15 where the vector difference is scaled $20 \times$ to demonstrate which direction and by how much the measured galvo errors exist. This also provides a visual demonstration of how a compensation reduces the individual point deviations.
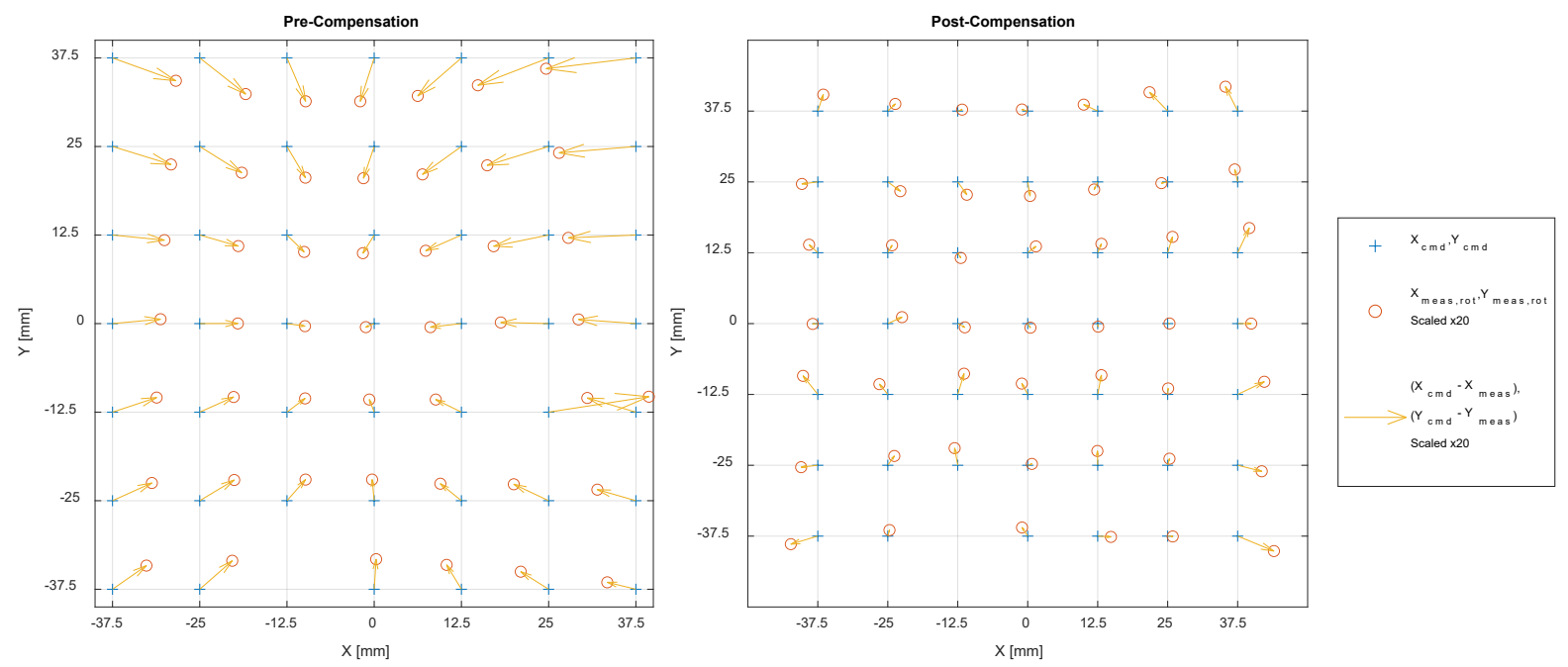

Fig. 15: Example commanded points $(+)$, measured points after rotation (o), and the vector difference between them (scaled $20 \times$ ) for pre-compensated and post-compensated measurements, demonstrating improvement in positioning accuracy post-compensated. 
While visually demonstrative, the vector error does not quantify how the galvo errors will 'dilate' or 'contract' a part build at a specific location. The deviation for each point is calculated as $E_{x}=\left|X_{\text {meas,rot }}\right|-\left|X_{\mathrm{cmd}}\right|$ in [mm], and similarly for $E_{\mathrm{y}}$. This definition using absolute values ensures that positive $\mathrm{E}_{\mathrm{x}}$ or $\mathrm{E}_{\mathrm{y}}$ results indicate 'dilation' of that point away from the origin, and negative errors indicate 'contraction' of that point towards the origin. The deviation values for all measured laser marks are plotted in Fig. 16 vs. the commanded position prior to and after a compensation. While still demonstrative of the deviationreducing effect after compensation, a singular metric should be defined for the positioning error that is the aggregate of individual deviations over the entire positioning range. Here we define this mean deviation as the mean deviation of all $\mathrm{E}_{\mathrm{x}}$ and $\mathrm{E}_{\mathrm{y}}$ values. For example, a positive average $\mathrm{E}_{\mathrm{x}}$ means the galvo tends to erroneously dilate in the $\mathrm{X}$ direction. The mean $\mathrm{E}_{\mathrm{x}}$ and $\mathrm{E}_{\mathrm{y}}$ are provided in the tables in Fig. 16.

Another metric is the relative positioning error. If the scaling (slope) of the calibration curves in Fig. 13 is erroneous, the scale of the $E_{x}$ and $E_{y}$ errors will tend to increase with positions further from the origin (i.e. the total field of points is dilated or contracted). Similarly, a part fabricated with smaller dimensions will tend to have small absolute geometric error compared to a large part. The deviation of a single point can be normalized by its relative distance from $(0,0)$, such as $\mathrm{E}_{\mathrm{x}} / \mathrm{X}_{\mathrm{cmd}}$ to form the relative deviation of that point. Taking the mean of all relative deviation values provides another singular metric for the total relative positioning error in each direction, and is demonstrated in the tables in Fig.16.
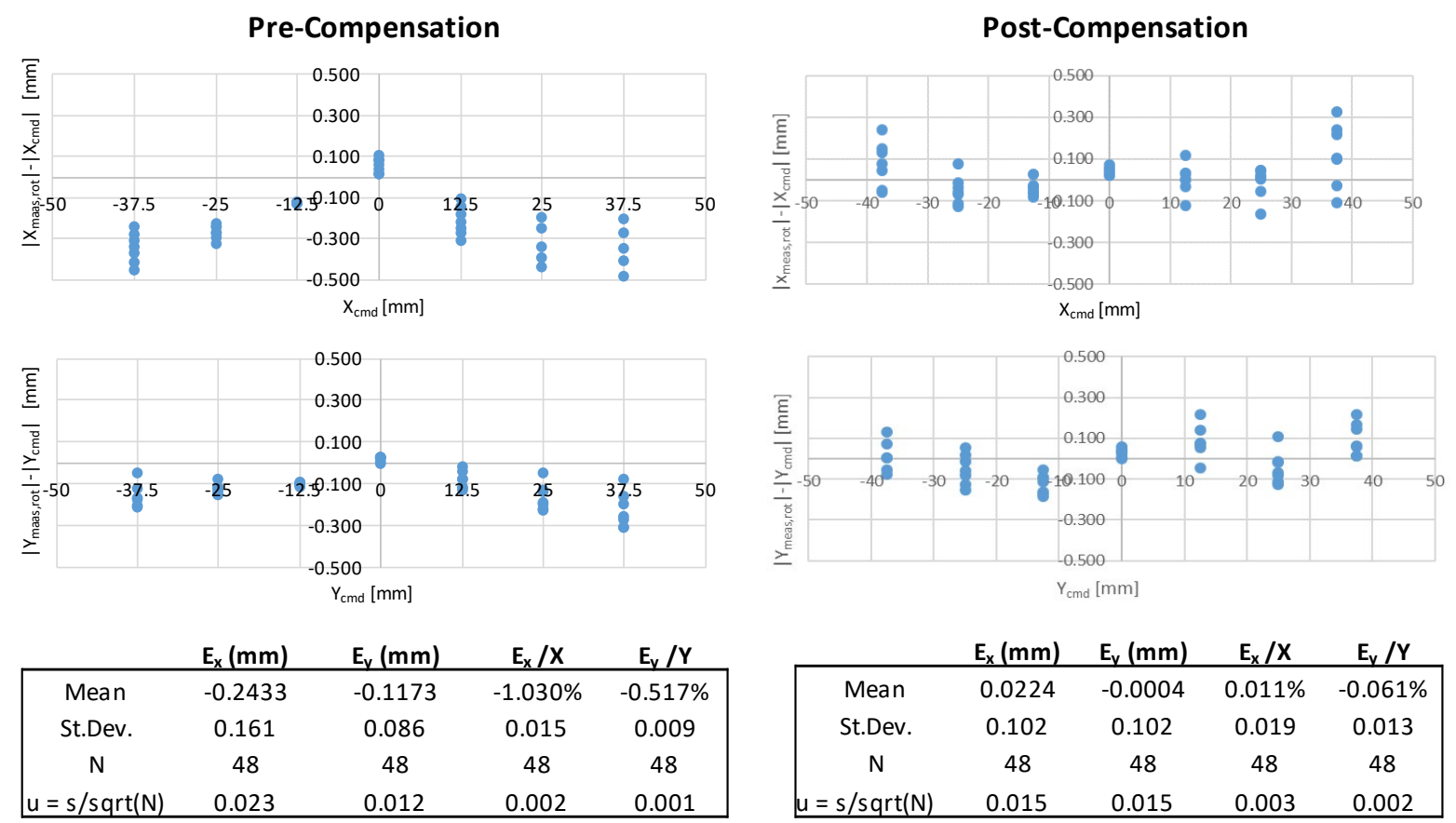

\begin{tabular}{|ccccc|}
\multicolumn{1}{c}{} & $\mathbf{E}_{\mathbf{x}}(\mathbf{m m})$ & $\mathbf{E}_{\mathrm{v}}(\mathbf{m m})$ & $\mathbf{E}_{\mathbf{x}} / \mathbf{X}$ & $\mathbf{E}_{\mathrm{v}} / \mathbf{Y}$ \\
\hline Mean & 0.0224 & -0.0004 & $0.011 \%$ & $-0.061 \%$ \\
St.Dev. & 0.102 & 0.102 & 0.019 & 0.013 \\
$\mathrm{~N}$ & 48 & 48 & 48 & 48 \\
$\mathrm{u}=\mathrm{s} /$ sqrt(N) & 0.015 & 0.015 & 0.003 & 0.002 \\
\hline
\end{tabular}

Fig. 16: Example errors $\mathrm{E}_{\mathrm{x}}$ and $\mathrm{E}_{\mathrm{y}}$ before compensation (left) and after compensation (right). After compensation various error metrics are reduced, as shown in the tables at the bottom of each figure. 
The relative deviations, $\mathrm{E}_{\mathrm{x}} / \mathrm{X}$ and $\mathrm{E}_{\mathrm{y}} / \mathrm{Y}$, defined at each measured point, are useful to describe how much the system may dilate or contract a part or feature built at that specific location, relative to the features planned or commanded dimensions. Larger features will incur larger deviation. For example, a thin-walled cylinder may incur a large absolute deviation of its outer diameter, but the wall thickness may incur a slight deviation due to its relatively smaller dimension. All the points that make up $\mathrm{E}_{\mathrm{x}} / \mathrm{X}$ and $\mathrm{E}_{\mathrm{y}} / \mathrm{Y}$ are analogous to a strain field. Positive relative deviation indicates feature dimensions are dilated in that direction and magnitude with respect to the planned geometry. Negative relative error indicates features are contracted in that direction.

The mean relative deviations give a single metric for how much the galvo errors generally dilate or contract parts in each direction. For example, in Fig.16, the post-compensated measurements resulted in general dilation of $0.019 \%$ in the X-direction and general contraction of $0.147 \%$ in the Y-direction. The relative positioning errors describe the mean positioning accuracy, which is less than the $0.1 \%$ desired accuracy for the AMMT in the Xdirection, and slightly greater than $0.1 \%$ in the Y-direction. However, these metrics incorporate the residual positioning errors prior to or after calibration and compensation, any unknown deterministic or stochastic errors, as well as measurement error associated with the mark and measure procedure. This is discussed further in Section 4: Measurement Uncertainty.

Another error metric described by Godineau et al. utilizes the cumulative distribution function (CDF) formed from the vector sum of the errors $\left(|E|=\sqrt{ } E_{x}{ }^{2}+E_{y}{ }^{2}\right)[8]$. This removes indication of how the galvo dilates or contracts points. However, the vector-sum CDF can tell how much of the measured field (in \%) has an error less than a certain value. For example, Fig. 17 shows that $90 \%$ of the scan area had $|\mathrm{E}|<0.41 \mathrm{~mm}$ pre- compensation, but $90 \%$ of the area had $|\mathrm{E}|<0.115 \mathrm{~mm}$ post- compensation. 

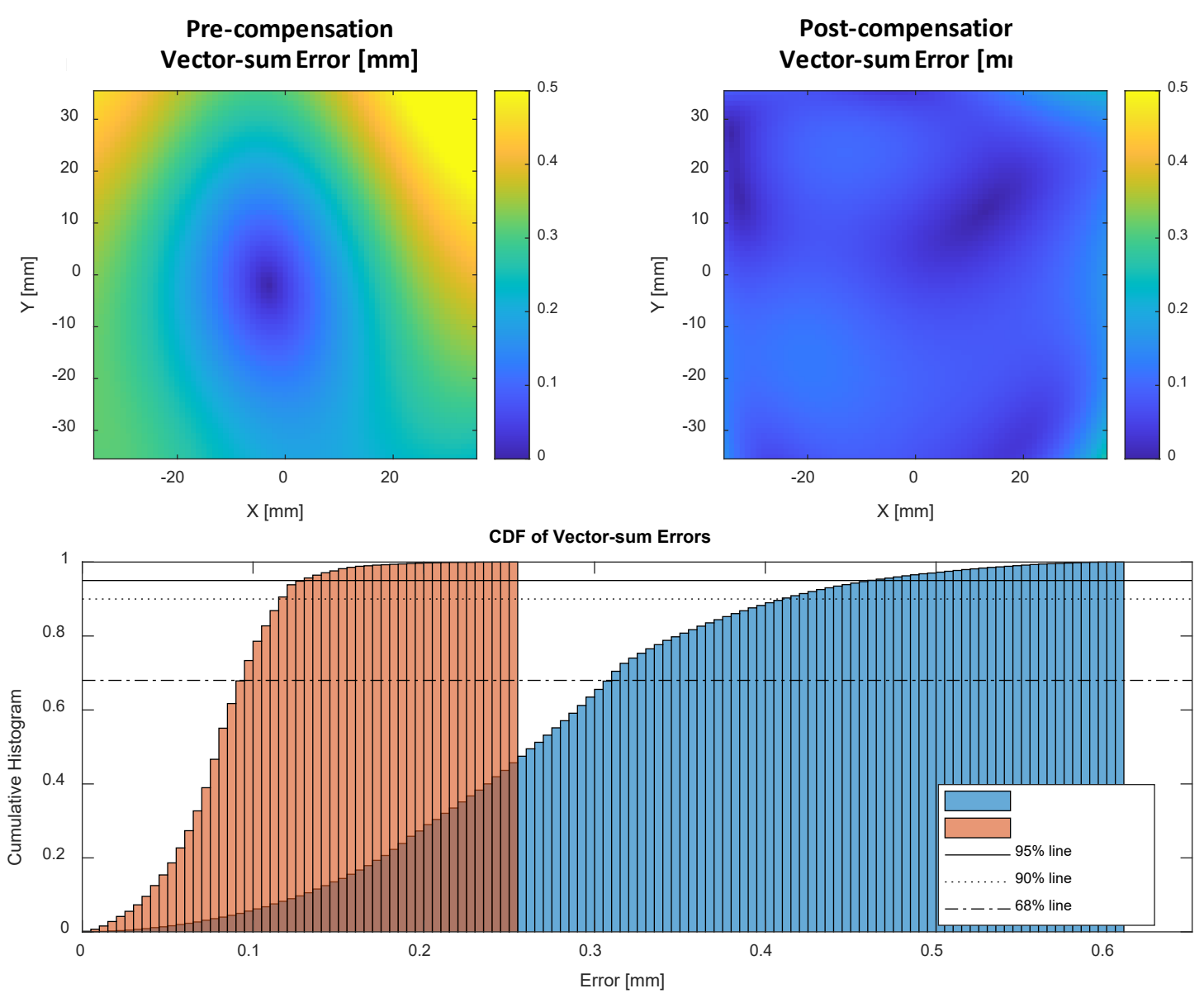

Fig. 17: Top: Example heat map of the vector sum error from pre- and post- compensation measurements, fit with a $4^{\text {rd }}$ order two-dimensional polynomial, and sub-sampled to a $1 \mathrm{~mm}$ grid. Bottom: Cumulative distributions of the sub-sampled vector-sum errors $|\mathrm{E}|$.

\section{Measurement Uncertainty}

While various error metrics have been described, this section focuses on the mean positioning error $\mathrm{E}_{\mathrm{x}}$, $\mathrm{E}_{\mathrm{y}}$ values in Fig.16. These are the measurands of interest, and the measurement uncertainty described here details how well these values are measured using the mark and measure method. A summarization of the uncertainty evaluation is provided in and summarized in Table 1 at the end of this section.

The measurement uncertainty of the mean error values are organized into three categories: 1) the physical marking setup and execution 2) the capture and measure of laser mark images and image processing 3 ) the calculation of error from the measured points. The following components of uncertainty are considered:

Z-positioning of plate describes the effect of positioning the calibration plate within the AMMT. Since the laser approaches the plate at an angle, any misalignment of the calibration plate surface with the expected build plane in the Z-direction will induce lateral positioning errors in $\mathrm{X}$ or $\mathrm{Y}$. This may stem from poor flatness of the calibration plate, non-parallelism 
with the build plane, or incorrectly positioning the calibration plate in the z-direction. The AMMT laser forms a maximum angle of $7.8^{\circ}$ from the surface normal within the calibrated area ( $\pm 37.5 \mathrm{~mm}$ from center) [9]. Therefore, a deviation in $\mathrm{Z}$ of the calibration plate by $\delta \mathrm{z}$ will elicit a maximum lateral deviation in $\mathrm{X}$ or $\mathrm{Y}$ by $\delta \mathrm{x}=\delta \mathrm{z} \cdot \tan \left(7.8^{\circ}\right)$. Although efforts are taken to parallelize and set the calibration plate to the specified build plane in the AMMT as described in Section 2, some positioning error may occur. The effect of this error on the galvo positioning will most likely not be random, but systematic. However, for simplicity, we assume a maximum deviation of $\delta \mathrm{z}< \pm 0.05 \mathrm{~mm}$ (Type $\mathrm{B}$ estimate), with assumed triangular distribution since $7.8^{\circ}$ is the maximum laser angle. Then, $\delta x$ or $\delta y$ will be $< \pm 0.007 \mathrm{~mm}$, and uncertainty $\mathrm{u}_{1}=\delta \mathrm{x} / \sqrt{ } 6[10]$.

Physical mark CoM matches laser spot center refers to the possibility that the CoM of the physical mark's image does not actually align with the center of the laser spot when it was created. This primarily stems from two factors: 1) The formation of the mark may be skewed by the physical marking process potentially stemming from molten pool sloshing, asymmetry of the laser energy profile, effect from the laser incident angle, positional noise/vibration of the laser spot, etc. 2) The image of the spot is not rotationally symmetric due to its inherent shape and structure, which elicits variability in the measured CoM. These factors are assumed to contribute variability less than $10 \%$ the average diameter of the marks, or roughly $\pm 0.01 \mathrm{~mm}$. Assuming normal distribution, Type $\mathrm{B}, \mathrm{u}_{2}=0.01 \mathrm{~mm}$.

Thermal errors incorporate any change in the aluminum plate dimensions due to thermal expansion between the marking process and measurement process. This may come from difference in ambient conditions, heat accumulation from handling, or heat accumulation during the marking process. Aluminum has a coefficient of thermal expansion (CTE) of approximately $24 \times 10^{-6} \mathrm{~m} / \mathrm{m} / \mathrm{K}$, meaning a $1{ }^{\circ} \mathrm{C}$ change in temperature would yield a $2.4 \mu \mathrm{m}$ change in the aluminum plate length. Careful handling and controlled lab environments can likely reduce temperature differences to $<1^{\circ} \mathrm{C}$. However, laser heating during the marking process does introduce a nontrivial amount of heat. Assuming all the laser energy during marking is absorbed and converted to heat energy in the plate, and assuming no heat loss (adiabatic), the laser pulses of $100 \mathrm{~ms}$ with $15 \mathrm{~W}$ amount to a total marking time of $4.8 \mathrm{~s}$, and total energy input of $72 \mathrm{~J}$. Plate mass (density $\times$ volume) is $\left(0.00271 \mathrm{~g} / \mathrm{mm}^{3}\right) \times(1.016 \mathrm{~mm} \times$ $100 \mathrm{~mm} \times 100 \mathrm{~mm})=27 \mathrm{~g}$. Specific heat for aluminum is approximately $0.9 \mathrm{~J} / \mathrm{g} / \mathrm{K}$, which yields a temperature rise $\Delta \mathrm{T}=\mathrm{q} / \mathrm{mc}=(72 \mathrm{~J}) /(27 \mathrm{~g}) /(0.9 \mathrm{~J} / \mathrm{g} / \mathrm{K}) \approx 3{ }^{\circ} \mathrm{C}$. This heat accumulation is non-equilibrium (transient), accumulates with each laser mark, and also very localized to the vicinity of each laser mark. The temperature rise at the instance of time during each mark will be $<3{ }^{\circ} \mathrm{C}$. We incorporate a very conservative estimate then that total temperature difference, from both environmental and laser marking effects, to be $\approx 3{ }^{\circ} \mathrm{C}$, or max thermal error on the $75 \mathrm{~mm}$ grid to be $\left(24 \times 10^{-6} \mathrm{~m} / \mathrm{m} / \mathrm{K}\right) \times(0.075 \mathrm{~m}) \times\left(3{ }^{\circ} \mathrm{C}\right)=5.4 \mu \mathrm{m}$. Assuming rectangular distribution, Type $B$, this yields $\mathrm{u}_{3}=5.4 \mu \mathrm{m} / \sqrt{ } 12=1.56 \mu \mathrm{m}$.

User selection of ROIs pertains to effect that the actual size and position of a user-selected ROI in ImageJ will affect the calculation of the CoM which determines that mark's location. To estimate this effect, 31 ROIs were made of one spot shown in Fig. 18, and the CoM calculated for each ROI. This resulted in standard deviation of $\sigma_{\mathrm{x}}=0.0085 \mathrm{~mm}$ and $\sigma_{\mathrm{y}}=0.0096 \mathrm{~mm}$. Since this was only conducted for one spot, and by one user, this 
uncertainty is assumed to be slightly greater than that measured in Fig. 18, or $0.010 \mathrm{~mm}$ (Type B, normal distribution).

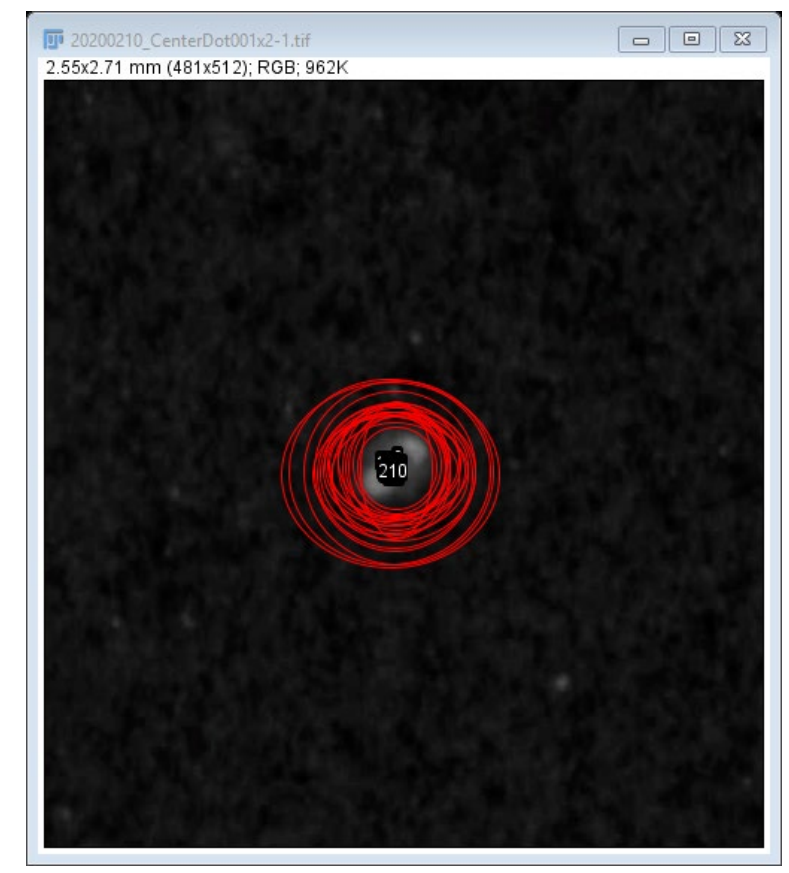

Fig. 18: Example ImageJ window showing 31 circular ROIs selected to test how varying the user-selected ROI size and location will affect the calculation of the spot's CoM.

Scaling factor is the length calibration of the flatbed scanner. The scanner length calibration was verified using a dot grid reference artefact with vendor-stated precision of $\pm 1 \mu \mathrm{m}$. At the $5.29 \mu \mathrm{m}$ per pixel resolution used in the galvo calibration measurements, the artefact's dot-to-dot spacing of $1 \mathrm{~mm}$ was repeatably measured to within $\pm 1 \mu \mathrm{m}$, indicating the scaling accuracy of the scanner itself is on the same order or better than the reference artefact's stated precision. A worst-case scenario assumes the scanner length calibration to be $<0.01 \%$. Over the $70 \mathrm{~mm}$ measured region, this results in $\mathrm{u}_{5}=0.007 \mathrm{~mm}$, with assumed normal probability distribution (Type B).

Standard uncertainty of the mean is provided in the tables in Fig.16. Since the number of samples, $N>20$, this is defined by $\mathrm{u}_{\mu}=\sigma / \sqrt{ } \mathrm{N}$; a Type A evaluation with normal distribution.

Combined standard uncertainty is calculated using the root-sum-square of the standard components of uncertainty assuming no correlations [10], or $\mathrm{u}_{\mathrm{c}}=\sqrt{\sum} \mathrm{u}_{\mathrm{i}}{ }^{2}$. The expanded uncertainty, $\mathrm{U}$, is the combined standard uncertainty multiplied by a coverage factor, $\mathrm{k}=2$, to define a level of confidence of approximately $95 \%$. 
Table 1: Measurement uncertainty budget for the mark and measure procedure. Uncertainty values are presented after any correction for non-normal (Gaussian) probability distribution.

\begin{tabular}{|l|c|c|c|c|}
\hline Category / Uncertainty Component & \multicolumn{2}{|c|}{ Uncertainty Value } & \multirow{2}{*}{ Tmm] } & Distribution \\
\hline Physical Measurement & $\mathbf{E}_{\mathbf{x}}$ & $\mathbf{E}_{\mathbf{y}}$ & & \\
\hline Z-positioning of plate, $\mathrm{u}_{1}$ & 0.0028 & 0.0028 & $\mathrm{~B}$ & Triangular \\
\hline Physical CoM matches laser center, $\mathrm{u}_{2}$ & 0.010 & 0.010 & $\mathrm{~B}$ & Normal \\
\hline Thermal errors, us & 0.0016 & 0.0016 & $\mathrm{~A}$ & Rectangular \\
\hline Scanned Image Capture & & & & \\
\hline User selection of ROIs, $\mathrm{u}_{4}$ & 0.010 & 0.010 & $\mathrm{~B}$ & Normal \\
\hline Scaling factor, us & 0.007 & 0.007 & $\mathrm{~B}$ & Normal \\
\hline Error Calculation (Post-compensation) & & & & \\
\hline Standard uncertainty of the mean $\sigma_{\mu}$ & 0.015 & 0.014 & $\mathrm{~A}$ & Normal \\
\hline Combined Standard Uncertainty, $\mathbf{u}_{\mathbf{c}}$ & 0.022 & 0.021 & & \\
\hline Expanded Uncertainty, U (k=2) & 0.044 & 0.043 & & \\
\hline
\end{tabular}

\section{References}

[1] Yeung H, Neira J, Lane B, Fox J, Lopez F (2016) Laser path planning and power control strategies for powder bed fusion systems. Proceedings of the 27th Annual International Solid Freeform Fabrication Symposium (Austin, TX), pp 113-127. Available at https://sffsymposium.engr.utexas.edu/sites/default/files/2016/008Yeung.pdf

[2] Yeung H, Lane BM, Donmez MA, Fox JC, Neira J (2018) Implementation of Advanced Laser Control Strategies for Powder Bed Fusion Systems. Procedia Manufacturing 26:871-879. https://doi.org/10.1016/j.promfg.2018.07.112

[3] Yeung H, Lane BM, Donmez MA, Moylan S (2020) In-situ calibration of laser/galvo scanning system using dimensional reference artefacts. CIRP Annals, $\mathrm{p} 4$.

[4] BiPM I, IFCC I, IUPAC I, ISO O (2012) The international vocabulary of metrologybasic and general concepts and associated terms (VIM). JCGM 200:2012.

[5] Land WS (2014) Effective calibration and implementation of galvanometer scanners as applied to direct metal laser sintering. Proceedings of the ASPE 2014 Spring Topical Meeting (American Society for Precision Engineering, Berkeley, CA), pp 151-156. 
[6] Halme R-J, Kumpulainen T, Tuokko R (2010) Enhancing laser scanner accuracy by grid correction. Micromachining and Microfabrication Process Technology XV (International Society for Optics and Photonics), Vol. 7590, p 759007. https://doi.org/10.1117/12.841741

[7] Lu Y (2017) Error Characterization of EOS M280 Additive Manufacturing Machine. Master of Science (Penn State University, State College, PA). Available at https://etda.libraries.psu.edu/catalog/12378yx1278

[8] Godineau K, Lavernhe S, Tournier C (2019) Calibration of galvanometric scan heads for additive manufacturing with machine assembly defects consideration. Additive Manufacturing 26:250-257. https://doi.org/10.1016/j.addma.2019.02.003

[9] Lane B, Yeung H (2019) Process monitoring dataset from the additive manufacturing metrology testbed (AMMT): "3d scan strategies." Journal of Research of the National Institute of Standards and Technology 124(124033). Available at https://doi.org/10.6028/jres. 124.033

[10] Taylor BN, Kuyatt CE (1994) Guidelines for evaluating and expressing the uncertainty of NIST measurement results. NIST Technical Note 1297.

\section{Appendix A: Supplemental Materials}

A Microsoft Excel spreadsheet (*.xlsx) file is available with two tabs/sheets that provide example 'pre-compensation' and 'post-compensation' measurement, calibration, plots, and measurement error calculations.

The file is titled "GalvoCalibrationCalculationSheet.xlsx", and is available at https://datapub.nist.gov/od/id/mds2-2257, with digital object identifier (DOI): https://doi.org/10.18434/M32257. 